\title{
Diffraction problem of scattering and propagation TM wave on pre-fractal impedance strips above shielded dielectric layer
}

\author{
Kateryna Nesvit \\ Department of Mathematical Physics and Computational Mathematics, Karazin Kharkiv National University, Ukraine \\ Corresponding authorE-mail: Kateryna.V.Nesvit@univer.kharkov.ua,nesvit.k@gmail.com
}

Copyright (C)2014 Kateryna Nesvit. This is an open access article distributed under the Creative Commons Attribution License, which permits unrestricted use, distribution, and reproduction in any medium, provided the original work is properly cited.

\begin{abstract}
In this paper a discrete mathematical model of the diffraction of a plane electromagnetic wave by pre-Cantor impedance strips on a shielded dielectric layer is developed. The TM wave case is considered. The mathematical model is based on the boundary singular integral equation (SIE) of the first kind with supplementary conditions and the Volterra integral equation (IE) of the second kind. Numerical experiments may be carried out based on this discrete mathematical model using the discrete singularities method (DSM).
\end{abstract}

Keywords: Diffraction, scattered, integral equation, impedance strips.

\section{Introduction}

Electromagnetic waves have two component E electric and $\mathrm{H}$ magnetic perpendicular to each other. When EM wave propagates with respect to direction of propagation we calculate $\mathrm{E}(\mathrm{x}, \mathrm{y}, \mathrm{z})$ or $\mathrm{H}(\mathrm{x}, \mathrm{y}, \mathrm{z})$. If the electric field of wave is purely transverse-right angle or 90 degree to the direction of propagation that wave called Transverse Electric (TE) wave. If the magnetic field of wave is purely transverse to the direction of propagation that wave called Transverse magnetic (TM) wave. And if both field are transverse that wave is TEM wave. In this paper the TM wave case is considered and without including of the surface waves.

Mathematical modeling of the interaction of electromagnetic radiation with plane-parallel electrodynamic structures is considered in many papers related to the solution of applied problems. These structures include pre-Cantor impedance gratings on a shielded dielectric layer. A similar problem in a periodic case with PEC strips and without the dielectric layer was studied in [1], [3], [5].

The model which is considered in this paper is an approximation of real fractal antenna in 2D. Fractal antennas are used in a variety of modern mobile devices due to their compact size and broadband properties, which have made them essential in wireless communication such as Bluetooth, Wi-Fi and GSM standards.

The overall aim of this work is to build a discrete mathematical model of the diffraction of a plane monochromatic TM wave on pre-Cantor [6] impedance strips, which are located above a dielectric layer on top of a flat screen reflector. The developed mathematical model of the considered diffraction problem is based on a SIE of the first kind with supplementary conditions and a Volterra IE of the second kind (see [1], [2]). They are discretized with the help of an efficient DSM. 


\section{TM wave diffraction problem}

Consider a TM plane wave of unitary amplitude falling from infinity onto the top of the diffraction structure (Fig. 1) at an angle $\alpha$ :

$u_{i n c}^{N}(y, z)=H_{x}(y, z)=e^{i k(y \sin \alpha-z \cos \alpha)}$.

Here $\alpha$ is an angle between positive direction of the $\mathrm{Z}$ axis and the propagation direction of a plane monochromatic wave, and $k$ is the absolute value of the wave vector $\vec{k}$.

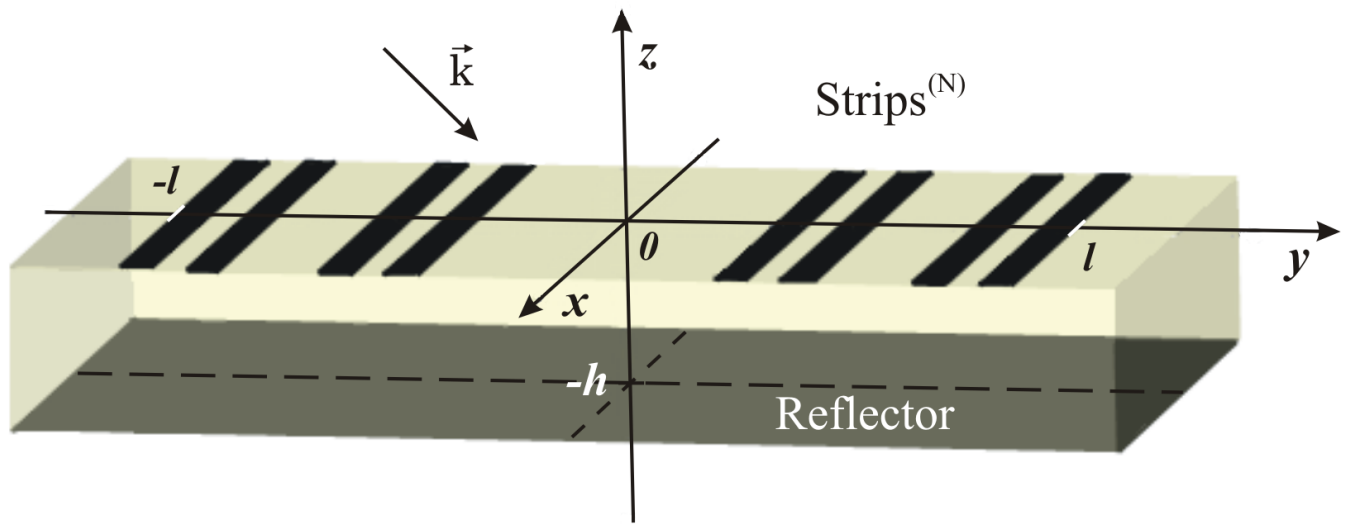

Figure 1: Schematic of the considered diffraction structure.

$$
\begin{aligned}
& \operatorname{Strips}^{(N)}=\left\{(x, y, z) \in \Re: x \in \Re, y \in S t_{l}^{(N)}, z=0\right\}, \\
& S t_{l}^{(N)}=\bigcup_{q=1}^{2^{N}}\left(a_{q}^{N}, b_{q}^{N}\right) .
\end{aligned}
$$

In this paper we consider a TM case where the unique independent component of the magnetic field $H_{x}=u(y, z)$ satisfies the two-dimensional Helmholtz equation above the flat screen reflector and without of the strips:

$\Delta H_{x}(y, z)+k^{2} H_{x}(y, z)=0, \quad k=\frac{\omega}{c}$.

The impedance boundary conditions of Shchukin - Leontovich on the strips and on the shield, as well as the Sommerfeld radiation conditions and the Meixner condition on the edges of the strips most be satisfied.

Components of the electromagnetic field vectors $\vec{E}(y, z)$ and $\vec{H}(y, z)$ are represented by $H_{x}(y, z)$ in the following form: $E_{x}(y, z)=H_{y}(y, z)=H_{z}(y, z)=0$,

$E_{y}(y, z)=-\frac{1}{i \omega \varepsilon} \frac{\partial}{\partial z} H_{x}(y, z), \quad E_{z}(y, z)=\frac{1}{i \omega \varepsilon} \frac{\partial}{\partial y} H_{x}(y, z)$.

For convenience let us switch to dimensionless coordinates:

$\varsigma=\frac{x}{l}, \xi=\frac{y}{l}, \zeta=\frac{z}{l}, \kappa=k l, d=\frac{h}{l}, \alpha_{q}^{N}=\frac{a_{q}^{N}}{l}, \beta_{q}^{N}=\frac{b_{q}^{N}}{l}$.

$S t^{(N)}=\bigcup_{q=1}^{2^{N}}\left(\alpha_{q}^{N}, \beta_{q}^{N}\right)$. 


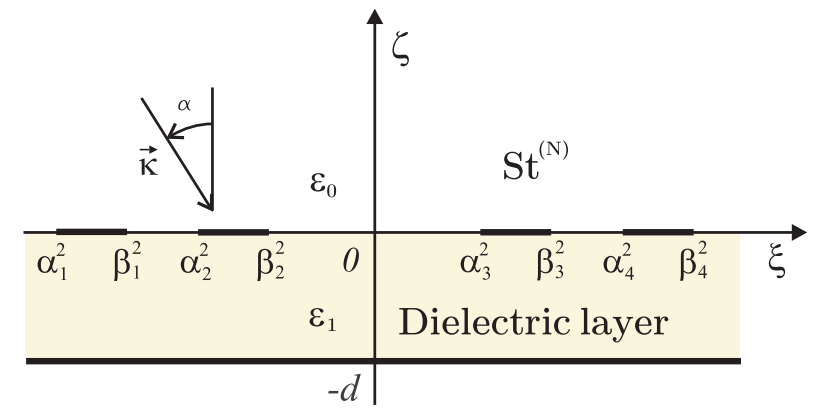

Figure 2: Cross-section of the diffraction structure in the $\zeta \xi$ plane.

The total field $u^{(N)}(\xi, \zeta)=H_{\varsigma}(\xi, \zeta)$ is considered to have the form

$u^{(N)}(\xi, \zeta)=\left\{\begin{array}{l}u_{0}^{N}(\xi, \zeta)+u_{+}^{N}(\xi, \zeta), \quad \zeta>0, \\ u_{0}^{N}(\xi, \zeta)+u_{-}^{N}(\xi, \zeta), \quad-d<\zeta<0,\end{array}\right.$

where $u_{0}^{N}(\xi, \zeta)$ is a known solution of the Helmholtz equation above a flat screen reflector for regions without of the strips. The functions $u_{+}^{N}(\xi, \zeta)$ and $u_{-}^{N}(\xi, \zeta)$ are to be determined.

The scattered field $u_{+}^{N}(\xi, \zeta)$ has the form

$u_{+}^{N}(\xi, \zeta)=\int_{-\infty}^{+\infty} C_{+}^{N}(\lambda) e^{i \lambda \xi-\gamma_{0}(\lambda) \zeta} d \lambda$

The radiation condition is satisfied if $\gamma_{0}(\lambda)=\sqrt{\lambda^{2}-\kappa^{2} \varepsilon_{0}}, \lambda \in \Re$ and is given by $\operatorname{Re}\left(\gamma_{0}(\lambda)\right) \geq 0, \operatorname{Im}\left(\gamma_{0}(\lambda)\right) \leq 0$.

The diffracted field $u_{-}^{N}(\xi, \zeta)$ can be written as

$u_{-}^{N}(\xi, \zeta)=\int_{-\infty}^{+\infty} C_{-}^{N}(\lambda) Z(\lambda, \zeta) e^{i \lambda \xi} d \lambda$

where function $Z(\lambda, \zeta)$ has the following properties:

$$
\begin{aligned}
& Z^{\prime}(\lambda, 0)=\gamma_{1}(\lambda), \\
& Z^{\prime}(\lambda,-d)-B_{2} Z(\lambda,-d)=0,
\end{aligned}
$$

and can be expressed as:

$Z(\lambda, \zeta)=\frac{B_{2} \operatorname{sh}\left(\gamma_{1}(\lambda)(\zeta+d)\right)+\gamma_{1}(\lambda) \operatorname{ch}\left(\gamma_{1}(\lambda)(\zeta+d)\right)}{B 2 \operatorname{ch}\left(\gamma_{1}(\lambda) d\right)+\gamma_{1}(\lambda) \operatorname{sh}\left(\gamma_{1}(\lambda) d\right)}$

The total field (7) should satisfy the following conditions:

- the Helmholtz equation (3) above the flat screen reflector for regions without of the strips;

- the boundary conditions on the strips:

$$
\left.\frac{\partial u_{+}^{N}(\xi, \zeta)}{\partial \zeta}\right|_{\zeta=+0}-\left.B_{1} u_{+}^{N}(\xi, \zeta)\right|_{\zeta=+0}=0, \quad \xi \in S t^{(N)}
$$


$-\left.\frac{\partial u_{-}^{N}(\xi, \zeta)}{\partial \zeta}\right|_{\zeta=-0}-\left.B_{2} u_{-}^{N}(\xi, \zeta)\right|_{\zeta=-0}=0, \quad \xi \in S t^{(N)}$,

and on the flat screen reflector:

$\left.\frac{\partial u_{-}^{N}(\xi, \zeta)}{\partial \zeta}\right|_{\zeta=-d+0}-\left.B_{2} u_{-}^{N}(\xi, \zeta)\right|_{\zeta=-d+0}=0, \quad \xi \in \Re$,

where $B_{1}, B_{2}$ are the impedances of metal,

$B_{1}=i \kappa \frac{Z_{c}}{Z_{0}^{1}}, B_{2}=i \kappa \frac{Z_{c}}{Z_{0}^{2}}, Z_{c}=\frac{1+i}{\sqrt{2}} \sqrt{\frac{\omega \mu_{c}}{\sigma_{c}}}, Z_{0}^{1}=\sqrt{\frac{\mu_{r} \mu_{0}}{\varepsilon_{r} \varepsilon_{0}}}, Z_{0}^{2}=\sqrt{\frac{\mu_{r} \mu_{1}}{\varepsilon_{r} \varepsilon_{1}}}$

- the conditions of conjugation in the slits $C S t^{(N)}=\Re \backslash S t^{(N)}$ :

$$
u^{(N)}(\xi,+0)=u^{(N)}(\xi,-0), \quad \xi \in C S t^{(N)},
$$

$$
\frac{1}{\varepsilon_{0}} \frac{\partial u^{(N)}}{\partial \zeta}(\xi,+0)=\frac{1}{\varepsilon_{1}} \frac{\partial u^{(N)}}{\partial \zeta}(\xi,-0), \quad \xi \in C S t^{(N)}
$$

- the Sommerfeld radiation conditions:

$$
u_{s c a t}^{(N)}=\underline{O}\left(\frac{1}{\sqrt{r}}\right), \quad \frac{\partial u_{s c a t}^{(N)}}{\partial r}-i \kappa u_{s c a t}^{(N)}=\bar{o}\left(\frac{1}{\sqrt{r}}\right), \quad r \rightarrow \infty ;
$$

- the Meixner edge conditions:

$$
E_{\varsigma}=\underline{O}\left(\rho^{\frac{\pi}{\varphi_{0}}}\right), \quad \rho \rightarrow 0, \quad \frac{\partial E_{\varsigma}}{\partial \rho}=\underline{O}\left(\rho^{\frac{\pi}{\varphi_{0}}-1}\right), \quad \rho \rightarrow 0,
$$

where $\rho$ is the distance from the edge to the point of observation.

\section{Mathematical model based on SIE}

Let us introduce two new unknown functions

$$
\begin{aligned}
& F_{1}^{N}(\eta)=\int_{-\infty}^{+\infty}\left(C_{+}^{N}(\lambda)-C_{-}^{N}(\lambda) Z(\lambda, 0)\right) i \lambda e^{i \lambda \eta} d \lambda, \eta \in \Re, \\
& F_{2}^{N}(\eta)=\int_{-\infty}^{+\infty}\left(\frac{\gamma_{0}(\lambda)}{\varepsilon_{0}} C_{+}^{N}(\lambda)+\frac{\gamma_{1}(\lambda)}{\varepsilon_{1}} C_{-}^{N}(\lambda) e^{i \lambda \eta} d \lambda, \eta \in \Re .\right.
\end{aligned}
$$

As shown in the monograph [1] and article [2], using the method of parametric representation of integral operators the boundary-value problem considering all conditions is reduced to a system of SIE of the first kind with 
supplementary conditions and a Volterra IE of the second kind:

$$
\left\{\begin{array}{l}
\frac{1}{\pi} \int_{S t^{(N)}} \frac{F_{1}^{N}(\eta)}{\eta-\xi} d \eta-B_{1} \int_{-\infty}^{\xi} F_{1}^{N}(\eta) d \eta+\left(\frac{\varepsilon_{0} \varepsilon_{1}}{\varepsilon_{0}+\varepsilon_{1}}\right)\left(B_{1}-B_{2}\right) \frac{1}{\pi} \int_{S t^{(N)}} \ln |\eta-\xi| F_{2}^{N}(\eta) d \eta+ \\
+\frac{1}{\pi} \int_{S t^{(N)}} Q_{1}^{N}(\eta, \xi) F_{1}^{N}(\eta) d \eta+\frac{1}{\pi} \int_{S t^{(N)}} Q_{2}^{N}(\eta, \xi) F_{2}^{N}(\eta) d \eta=f_{1}^{N}(\xi), \quad \xi \in S t^{(N)}, \\
\int_{S t_{p}^{(N)}} F_{1}^{N}(\eta) d \eta=0, p=\overline{1,2^{N}}, \quad S t_{q}^{(N)}=\left(\alpha_{q}^{N}, \beta_{q}^{N}\right), \\
F_{2}^{N}(\xi)+B_{1} \int_{-\infty}^{\xi} F_{1}^{N}(\eta) d \eta-\left(\frac{\varepsilon_{0} \varepsilon_{1}}{\varepsilon_{0}+\varepsilon_{1}}\right)\left(\frac{B_{1}}{\varepsilon_{0}}+\frac{B_{2}}{\varepsilon_{1}}\right) \frac{1}{\pi} \int_{S t^{(N)}} \ln |\eta-\xi| F_{2}^{N}(\eta) d \eta+ \\
+\frac{1}{\pi} \int_{S t^{(N)}} Q_{3}^{N}(\eta, \xi) F_{1}^{N}(\eta) d \eta+\frac{1}{\pi} \int_{S t^{(N)}} Q_{4}^{N}(\eta, \xi) F_{2}^{N}(\eta) d \eta=f_{2}^{N}(\xi), \quad \xi \in S t^{(N)},
\end{array}\right.
$$

where $Q_{i}^{N}(\eta, \xi), i=\overline{1,4}, \quad f_{m}^{N}(\xi), m=1,2$ are known functions.

To reduce equation (21) on $S t^{(N)}$ to equations on a set of intervals $S t_{q}^{(N)}$ we introduce restrictive conditions to the functions:

$F_{i, q}^{N}(\eta)=\left.F_{i}^{N}(\eta)\right|_{\eta \in S t_{q}^{(N)}}, \quad F_{2, p}^{N}(\xi)=\left.F_{2}^{N}(\xi)\right|_{\xi \in S t_{p}^{(N)}}, \quad f_{i, p}^{N}(\xi)=\left.f_{i}^{N}(\xi)\right|_{\xi \in S t_{p}^{(N)}}$,

where $i=1,2 ; p, q=\overline{1,2^{N}}$.

In this case, the Meixner edge condition will be satisfied if the unknown functions (20) are represented in the form of:

$F_{i, q}^{N}(\eta)=\frac{v_{i, q}^{N}(\eta)}{\sqrt{\left(\beta_{q}^{N}-\eta\right)\left(\eta-\alpha_{q}^{N}\right)}}, \quad F_{2, p}^{N}(\xi)=\frac{v_{2, p}^{N}(\xi)}{\sqrt{\left(\beta_{q}^{N}-\xi\right)\left(\xi-\alpha_{q}^{N}\right)}}$

where $\eta \in S t_{q}^{(N)}, \xi \in S t_{p}^{(N)}, i=1,2, p=\overline{1,2^{N}}$.

By choosing a normalized interval $(-1,1)$ the variables transform accordingly:

$$
\begin{aligned}
& g_{q}^{(N)}:(-1,1) \rightarrow\left(\alpha_{q}^{N}, \beta_{q}^{N}\right): \\
& t \rightarrow g_{q}^{(N)}(t)=\frac{\beta_{q}^{N}-\alpha_{q}^{N}}{2} t+\frac{\beta_{q}^{N}+\alpha_{q}^{N}}{2}, \\
& \eta=g_{q}^{(N)}(t), \quad \xi=g_{p}^{(N)}\left(t_{0}\right), \quad|t|<1, \quad\left|t_{0}\right|<1, \\
& \eta \in S t_{q}^{N}, \quad \xi \in S t_{q}^{N}, \quad p, q=\overline{1,2^{N}} .
\end{aligned}
$$

Considering (22)-(24) and excluding singular and logarithmic singularities at $p=q$ from the system of IEs (21), we obtain a system of SIEs of the 1-st kind with supplementary conditions, and a Volterra IEs of the 2-nd kind 
with singular and logarithmic singularities on a normalized interval for $p=\overline{1,2^{N}}$ :

$$
\begin{aligned}
& \frac{2}{\beta_{p}^{N}-\alpha_{p}^{N}} \frac{1}{\pi} \int_{-1}^{1} \frac{v_{1, p}^{N}\left(g_{p}^{(N)}(t)\right)}{t-t_{0}} \frac{d t}{\sqrt{1-t^{2}}}-B_{1} \int_{-1}^{t_{0}} v_{1, p}^{N}\left(g_{p}^{(N)}(t)\right) \frac{d t}{\sqrt{1-t^{2}}}+ \\
& +\left(\frac{\varepsilon_{0} \varepsilon_{1}}{\varepsilon_{0}+\varepsilon_{1}}\right)\left(B_{1}-B_{2}\right) \frac{1}{\pi} \int_{-1}^{1} \ln \left|t-t_{0}\right| v_{2, p}^{N}\left(g_{p}^{(N)}(t)\right) \frac{d t}{\sqrt{1-t^{2}}}+ \\
& +\frac{1}{\pi} \sum_{q=1}^{2^{N}} \int_{-1}^{1} K_{1, q, p}^{N}\left(g_{q}^{(N)}(t), g_{p}^{(N)}\left(t_{0}\right)\right) v_{1, q}^{N}\left(g_{q}^{(N)}(t)\right) \frac{d t}{\sqrt{1-t^{2}}}+ \\
& +\frac{1}{\pi} \sum_{q=1}^{2^{N}} \int_{-1}^{1} K_{2, q, p}^{N}\left(g_{q}^{(N)}(t), g_{p}^{(N)}\left(t_{0}\right)\right) v_{2, q}^{N}\left(g_{q}^{(N)}(t)\right) \frac{d t}{\sqrt{1-t^{2}}}=f_{1, p}^{N}\left(g_{p}^{(N)}\left(t_{0}\right)\right) \\
& \int_{-1}^{1} v_{1, p}^{N}\left(g_{p}^{(N)}(t)\right) \frac{d t}{\sqrt{1-t^{2}}}=0, \\
& \frac{2}{\beta_{p}^{N}-\alpha_{p}^{N}} \frac{v_{2, p}^{N}\left(g_{p}^{(N)}\left(t_{0}\right)\right)}{\sqrt{1-t_{0}^{2}}}+B_{1} \int_{-1}^{t_{0}} v_{1, p}^{N}\left(g_{p}^{(N)}(t)\right) \frac{d t}{\sqrt{1-t^{2}}}- \\
& -\left(\frac{\varepsilon_{0} \varepsilon_{1}}{\varepsilon_{0}+\varepsilon_{1}}\right)\left(\frac{B_{1}}{\varepsilon_{0}}+\frac{B_{2}}{\varepsilon_{1}}\right) \frac{1}{\pi} \int_{-1}^{1} \ln \left|t-t_{0}\right| v_{2, p}^{N}\left(g_{p}^{(N)}(t)\right) \frac{d t}{\sqrt{1-t^{2}}}+ \\
& +\frac{1}{\pi} \sum_{q=1}^{2^{N}} \int_{-1}^{1} K_{3, q, p}^{N}\left(g_{q}^{(N)}(t), g_{p}^{(N)}\left(t_{0}\right)\right) v_{1, q}^{N}\left(g_{q}^{(N)}(t)\right) \frac{d t}{\sqrt{1-t^{2}}}+ \\
& +\frac{1}{\pi} \sum_{q=1}^{2^{N}} \int_{-1}^{1} K_{4, q, p}^{N}\left(g_{q}^{(N)}(t), g_{p}^{(N)}\left(t_{0}\right)\right) v_{2, q}^{N}\left(g_{q}^{(N)}(t)\right) \frac{d t}{\sqrt{1-t^{2}}}=f_{2, p}^{N}\left(g_{p}^{(N)}\left(t_{0}\right)\right)
\end{aligned}
$$

where $K_{i, q, p}^{N}\left(g_{q}^{(N)}(t), g_{p}^{(N)}\left(t_{0}\right)\right), i=\overline{1,4} ; f_{m}^{N}\left(g_{p}^{(N)}\left(t_{0}\right)\right), m=1,2$ are known functions.

\section{Discrete mathematical model}

The unknown functions

$v_{i, q}^{N}\left(g_{q}^{(N)}(t)\right), i=1,2$,

and smooth functions

$K_{i, q, p}^{N}\left(g_{q}^{(N)}(t), g_{p}^{(N)}\left(t_{0}\right)\right), \quad i=\overline{1,4} \quad f_{m}^{N}\left(g_{p}^{(N)}\left(t_{0}\right)\right), \quad m=1,2$,

are interpolated by a Lagrange polynomial of (n-1)-th degree in the nodes which are the nulls of Chebyshev polynomials of the $1^{\text {st }}$ kind:

$t_{k}^{n}=\cos \left(\frac{2 k-1}{2 n} \pi\right), \quad k=\overline{1, n}$.

From the mathematical model in (25) we have obtained a system for approximate solutions. In the next step, using quadrature formulas [4] for integrals with singular and logarithmic singularities and integrals of smooth functions, we derive a system of linear algebraic equations (SLAE) for the values of unknown functions in the node points:

$v_{i, q}^{N,(n-1)}\left(g_{q}^{(N)}\left(t_{k}^{n}\right)\right), \quad i=1,2$. 
At collocated points we select another set of Chebychev nodes:

$t_{0 j}^{n}=\cos \frac{j}{n} \pi, \quad j=\overline{1, n-1}$.

And therefore we can write down a SLAE for the solution of which will give us the desired unknown functions in the discrete form:

$$
\begin{aligned}
& \frac{2}{\beta_{p}^{N}-\alpha_{p}^{N}} \frac{1}{n} \sum_{k=1}^{n} \frac{v_{1, p}^{N,(n-1)}\left(g_{p}^{(N)}\left(t_{k}^{n}\right)\right)}{t_{k}^{n}-t_{o j}^{n}}- \\
& -B_{1} \frac{1}{n} \sum_{k=1}^{n} v_{1, p}^{N,(n-1)}\left(g_{p}^{(N)}\left(t_{k}^{n}\right)\right)\left[\pi-\arccos \left(t_{o j}^{n}\right)-2 \sum_{p=1}^{n-1} \frac{T_{p}\left(t_{k}^{n}\right)}{p} U_{p-1}\left(t_{0 j}^{n}\right) \sqrt{1-t_{0 j}^{2}}\right]- \\
& -\left(\frac{\varepsilon_{0} \varepsilon_{1}}{\varepsilon_{0}+\varepsilon_{1}}\right)\left(B_{1}-B_{2}\right) \frac{1}{n} \sum_{k=1}^{n} v_{2, p}^{N,(n-1)}\left(g_{p}^{(N)}\left(t_{k}^{n}\right)\right)\left[\ln 2+2 \sum_{r=1}^{n-1} T_{r}\left(t_{0 j}^{n}\right) \frac{T_{r}\left(t_{k}^{n}\right)}{r}\right]+ \\
& +\frac{1}{n} \sum_{q=1}^{2^{N}} \sum_{k=1}^{n} K_{1, q, p}^{N,(n-1),(n-2)}\left(g_{q}^{(N)}\left(t_{k}^{n}\right), g_{p}^{(N)}\left(t_{0 j}^{n}\right)\right) v_{1, q}^{N,(n-1)}\left(g_{q}^{(N)}\left(t_{k}^{n}\right)\right)+ \\
& +\frac{1}{n} \sum_{q=1}^{2^{N}} \sum_{k=1}^{n} K_{2, q, p}^{N,(n-1),(n-2)}\left(g_{q}^{(N)}\left(t_{k}^{n}\right), g_{p}^{(N)}\left(t_{0 j}^{n}\right)\right) v_{2, q}^{N,(n-1)}\left(g_{q}^{(N)}\left(t_{k}^{n}\right)\right)=f_{1, p}^{N,(n-2)}\left(g_{p}^{(N)}\left(t_{0 j}^{n}\right)\right), \\
& \frac{1}{n} \sum_{k=1}^{n} v_{1, p}^{N,(n-1)}\left(g_{p}^{(N)}\left(t_{k}^{n}\right)\right)=0 \\
& \frac{2}{\beta_{p}^{N}-\alpha_{p}^{N}} \frac{v_{2, p}^{N,(n-1)}\left(g_{p}^{(N)}\left(t_{0 j}^{n}\right)\right)}{\sqrt{1-\left(t_{0 j}^{n}\right)^{2}}}+B_{1} \frac{1}{n} \sum_{k=1}^{n} v_{1, p}^{N,(n-1)}\left(g_{p}^{(N)}\left(t_{k}^{n}\right)\right) \times \\
& \times\left[\pi-\arccos \left(t_{o j}^{n}\right)-2 \sum_{p=1}^{n-1} \frac{T_{p}\left(t_{k}^{n}\right)}{p} U_{p-1}\left(t_{0 j}^{n}\right) \sqrt{1-t_{0 j}^{2}}\right]- \\
& -\left(\frac{\varepsilon_{0} \varepsilon_{1}}{\varepsilon_{0}+\varepsilon_{1}}\right)\left(\frac{B_{1}}{\varepsilon_{0}}+\frac{B_{2}}{\varepsilon_{1}}\right) \frac{1}{n} \sum_{k=1}^{n} v_{2, p}^{N,(n-1)}\left(g_{p}^{(N)}\left(t_{k}^{n}\right)\right)\left[\ln 2+2 \sum_{r=1}^{n-1} T_{r}\left(t_{0 j}^{n}\right) \frac{T_{r}\left(t_{k}^{n}\right)}{r}\right]+ \\
& +\frac{1}{n} \sum_{q=1}^{2^{N}} \sum_{k=1}^{n} K_{3, q, p}^{N,(n-1),(n-2)}\left(g_{q}^{(N)}\left(t_{k}^{n}\right), g_{p}^{(N)}\left(t_{0 j}^{n}\right)\right) v_{1, q}^{N,(n-1)}\left(g_{q}^{(N)}\left(t_{k}^{n}\right)\right)+ \\
& +\frac{1}{n} \sum_{q=1}^{2^{N}} \sum_{k=1}^{n} K_{4, q, p}^{N,(n-1),(n-2)}\left(g_{q}^{(N)}\left(t_{k}^{n}\right), g_{p}^{(N)}\left(t_{0 j}^{n}\right)\right) v_{2, q}^{N,(n-1)}\left(g_{q}^{(N)}\left(t_{k}^{n}\right)\right)=f_{2, p}^{N,(n-2)}\left(g_{p}^{(N)}\left(t_{0 j}^{n}\right)\right),
\end{aligned}
$$

where $K_{i, q, p}^{N,(n-1),(n-2)}\left(g_{q}^{(N)}\left(t_{k}^{n}\right), g_{p}^{(N)}\left(t_{0 j}^{n}\right)\right), i=\overline{1,4} ; \quad f_{m}^{N,(n-2)}\left(g_{p}^{(N)}\left(t_{0 j}^{n}\right)\right), m=1,2$ are smooth known functions.

\section{Conclusion}

The discrete mathematical model of the diffraction problem of a plane monochromatic TM wave on pre-Cantor impedance strips, which are located above a dielectric layer on top of a flat screen reflector, has been developed with the help of an efficient DSM and special quadrature formulas with the nodes in the nulls of Chebyshev polynomials. As a next step we plan to perform numerical simulations and analyze the performance of this approach for both Eand H-polarization cases. 


\section{Acknowledgements}

The author is grateful to the supervisor, Prof. Yu. V. Gandel for many extremely fruitful conversations, invaluable assistance and useful.

\section{References}

[1] Yu. V. Gandel, V. D. Dushkin, Mathematical models of two-dimensional diffraction problems: Singular integral equations and numerical discrete singularities method (in Russian), Monograph, Kharkov: Academy of Internal Defence of the MIA of Ukraine, (2012).

[2] Yu. V. Gandel, V. L. Sidelnikov, Method of integral equations in third boundary-value diffraction problem on boundary grating above a flat screen reflector (in Russian). Differential equations. Vol. 35, No. 9, (1999), pp. $1155-1161$.

[3] K. V. Nesvit, Discrete mathematical model of diffraction on periodic pre-Cantor gratings with shield and numerical experiment. Bulletin of Karazin Kharkiv National University 1037 Series "Mathematical modeling. Information Technology. Automated Control Systems, Issue 20, (2012), pp. 146-157.

[4] Yu. V. Gandel, Introduction to methods of evaluation of singular and hypersingular integrals (in Russian). Textbook, Kharkov, Ukraine, (2002).

[5] I. K. Lifanov, The method of singular integral equations and numerical experiment (in Russian), TOO "Janus", Moscow, Russia, (1995).

[6] B. B. Mandelbrot, The Fractal Geometry of Nature, W. H. Freeman and Company, New York, (1983). 